\title{
Evaluation of a Minimally Invasive Image-Guided Surgery System for Hepatic Ablation Procedures
}

\author{
Chet W. Hammill, MD ${ }^{1,5}$, Logan W. Clements, $\mathrm{PhD}^{2}$, James D. Stefansic, $\mathrm{PhD}^{3}$, Ronald F. \\ Wolf, MD ${ }^{1,5}$, Paul D. Hansen, MD ${ }^{1,5}$, and David A. Gerber, MD $^{4}$ \\ ${ }_{1}^{1}$ Providence Portland Medical Center, Portland, OR, USA \\ ${ }^{2}$ Vanderbilt University, Nashville, TN, USA \\ ${ }^{3}$ Pathfinder Technologies Inc, Nashville, TN, USA \\ ${ }^{4}$ University of North Carolina School of Medicine, Chapel Hill, NC, USA \\ ${ }^{5}$ The Oregon Clinic, Portland, OR, USA
}

\begin{abstract}
Background-The Explorer Minimally Invasive Liver (MIL) system uses imaging to create a 3dimensional model of the liver. Intraoperatively, the system displays the position of instruments relative to the virtual liver. A prospective clinical study compared it with intraoperative ultrasound (iUS) in laparoscopic liver ablations.
\end{abstract}

\begin{abstract}
Methods-Patients undergoing ablations were accrued from 2 clinical sites. During the procedures, probes were positioned in the standard fashion using iUS. The position was synchronously recorded using the Explorer system. The distances from the probe tip to the tumor boundary and center were measured on the ultrasound image and in the corresponding virtual image captured by the Explorer system.
\end{abstract}

Results-Data were obtained on the placement of 47 ablation probes during 27 procedures. The absolute difference between iUS and the Explorer system for the probe tip to tumor boundary distance was $5.5 \pm 5.6 \mathrm{~mm}$, not a statistically significant difference. The absolute difference for probe tip to tumor center distance was $8.6 \pm 7.0 \mathrm{~mm}$, not statistically different from $5 \mathrm{~mm}$.

Discussion-The initial clinical experience with the Explorer MIL system shows a strong correlation with iUS for the positioning of ablation probes. The Explorer MIL system is a promising tool to provide supplemental guidance information during laparoscopic liver ablation procedures.

\section{(C) The Author(s) 2013}

Corresponding Author: Chet W. Hammill, Liver and Pancreas Surgery Program, Providence Portland Medical Center, 4805 NE Glisan Street, Suite 6N-60, Portland, OR 97213, USA. chammill@orclinic.com.

* The Euclidean distance between point $\mathrm{a}\left(x_{\mathrm{a}}, y_{\mathrm{a}}, z_{\mathrm{a}}\right)$ and point $\mathrm{b}\left(x_{\mathrm{b}}, y_{\mathrm{b}}, z_{\mathrm{b}}\right)$ is defined by the following equation:

$\sqrt{\left(x_{\mathrm{a}}-x_{\mathrm{b}}\right)^{2}+\left(y_{\mathrm{a}}-y_{\mathrm{b}}\right)^{2}+\left(z_{\mathrm{a}}-z_{\mathrm{b}}\right)^{2}}$.

Declaration of Conflicting Interests

The author(s) declared the following potential conflicts of interest with respect to the research, authorship, and/or publication of this article: The research was funded by Pathfinder Technologies and several of the authors were employees of or consultants for Pathfinder Technologies. 


\section{Keywords}

laparosopic liver surgery; ablation; image-guided surgery; laparoscopic ultrasound

\section{Introduction}

Three-dimensional (3D) image-guidance systems use preoperative imaging to create a 3D virtual model of organs. Intraoperatively, the systems track instruments and display their position relative to the organ and any structures of interest. The use of these systems for open hepatic resection and ablation procedures has been documented in the literature. ${ }^{1,2}$ With increasing comorbidities associated with select patient populations (eg, cirrhosis) there has been an evolution of minimally invasive approaches to treat hepatic tumors. ${ }^{3-6}$

Laparoscopic procedures require improved visualization to compensate for the lack of tactile feedback and these procedures could benefit from the utilization of image-guidance technology. Currently, the majority of operative hepatic ablation procedures are performed using intraoperative ultrasound (iUS), which requires considerable expertise and training. The incorporation of 3D image-guidance is a potentially valuable adjunct. One area in particular where this technology could be useful is in the targeting of lesions in cirrhotic patients where the quality of iUS imaging is severely compromised by the parenchymal changes throughout the liver. ${ }^{7}$

The Explorer Minimally Invasive Liver (MIL) system (Pathfinder Technologies, Nashville, $\mathrm{TN}$ ) is an experimental 3D image-guidance system intended to be used in conjunction with iUS during laparoscopic liver ablations. The system consists of several components. The Scout Liver planning software (Pathfinder Technologies, Nashville, TN) generates 3D anatomical models of the liver, vessels, and tumors from preoperative computed tomography (CT) or magnetic resonance imaging (MRI) scans. The tracking system cart has a monitor, for displaying preoperative CT or MRI images and the 3D virtual models of the liver; an infrared light source; and a pair of infrared cameras for optical tracking of the Pathfinder Tracer arrays, described below. The tracked laparoscopic stylus is a calibrated instrument that is used to synchronize the position of the liver with the virtual model, a process called registration. Finally, Pathfinder Tracer arrays are adapters that can be attached to different ablation probes. The arrays reflect infrared light in a predetermined pattern that can be seen by the cameras and used to determine the position and configuration of the attached ablation probe. A schematic of the device hardware and software interface are shown in Figure 1.

As a first step toward the use of the Explorer MIL system during laparoscopic ablation procedures, this prospective clinical study compares the MIL system with iUS. During the performance of laparoscopic liver ablations, the probes were placed in the standard fashion using iUS. Just prior to initiating the ablation, the position of the probe was recorded using the Explorer MIL system. The purpose of the study was to compare the difference between the measurements made using the Explorer MIL system and those made using iUS. 


\section{Methods}

\section{Patient Population}

From November 2010 to February 2012, subjects undergoing laparoscopic ablation procedures $(\mathrm{N}=27)$ were accrued at Providence Portland Medical Center in Portland, Oregon and the University of North Carolina Hospitals in Chapel Hill, North Carolina. The demographics of the included patients are summarized in Table 1. Ablations were performed using either the Angiodynamics StarBurst Xli-enhanced radiofrequency ablation (RFA) system or the Coviden Evident microwave ablation (MWA) system based on surgeon/center practice. Images and schematics of these 2 ablation systems are shown in Figure 2.

\section{Presurgical Planning}

Prior to performing the laparoscopic ablation procedure, the preoperative surgical planning software (Scout Liver) was used to generate 3D models of the liver, tumors, and vascular structures. Standard anatomical features were highlighted on the liver surface for use in the intraoperative registration process.

\section{Surgical Procedure}

The laparoscopic ablation procedures were performed per institutional protocol with one modification. Immediately prior to the placement of the ablation probe, a Pathfinder registration was performed. Registration is the synchronization of the liver position with the 3D virtual model and involves tracing preselected anatomical features on the surface of the liver with the tracked laparoscopic stylus (Figure 1). ${ }^{8}$ The surgeon then qualitatively evaluated the registration accuracy by moving the stylus over the liver surface and/or touching anatomical landmarks to verify that the position corresponded with that seen on the virtual model.

The ablation probe, with the attached Pathfinder Tracer array, was then positioned using iUS guidance. To be clear, the Explorer MIL system was not used to assist in the placement of the probe. Immediately prior to the initiation of ablation, the location and trajectory of the ablation probe were recorded using the Explorer MIL system. In addition, iUS images showing the final probe placement were obtained. A screenshot of the Explorer MIL system display immediately prior to recording the ablation probe location is shown in Figure 3. In cases where multiple tumors were treated, data were collected for each ablation probe placement.

\section{Postprocedure Analysis and Statistical Considerations}

Data analysis acquired in the clinical evaluation of the Explorer MIL system was performed by comparing guidance information obtained from the system to iUS. Two measurements were used to compare the 2 modalities: ( $a$ ) the Euclidean distance* between the tip of the ablation probe and the center of the tumor and $(b)$ the closest point distance between the probe tip and the tumor boundary. An example of the distance measurements obtained from the 2 modalities is shown in Figure 4. 
Descriptive statistics, including means, standard deviations, medians, confidence intervals, and ranges were calculated using the measurements obtained from both modalities. Statistical comparison was performed with paired $t$ tests or the Wilcoxon signed rank test depending on how well the data met the corresponding assumptions. In cases where it was unclear whether the assumptions were met, both parametric and nonparametric tests were performed.

\section{Results}

A summary of the data analysis comparing the Explorer MIL system with iUS is shown in Table 2 . To more accurately represent the relationship between the tip of the ablation probe and the tumor boundary, the distance was assigned a positive value if the tip of the probe was located outside the tumor and a negative value if the tip was located inside the tumor. The absolute difference between the distance measurements made for the 2 modalities were used to calculate the statistical parameters reported in the summary.

The results of the paired statistical tests performed for the measurements made with the 2 modalities are shown in Table 3. Given that the tip to tumor boundary data did not pass the normality test, the statistical comparison was performed using a Wilcoxon signed rank test where the median difference between the populations was hypothesized to be $0 \mathrm{~mm}$. The results of the Wilcoxon signed rank test indicate that there is no statistically significant difference between the instrument tip to tumor boundary measurements made using the Explorer MIL system and iUS. The paired $t$ test was used for comparison of the tip-to-tumor center data since they were normally distributed. When the difference in means was hypothesized to be $0 \mathrm{~mm}$, there was a statistically significant difference between the tip-tocenter distance measurements obtained from the 2 modalities. However, when the difference in means was hypothesized to be $5 \mathrm{~mm}$, the paired $t$ test did not find a statistically significant difference between the tip-to-center measurements. Given that the data for the tip to center distance measurements passed normality tests, a power calculation can be performed for the statistical test. The standard deviation for the ablation tip-to-lesion center distance measurements indicates that the paired $t$ test was capable of detecting a difference of $3.8 \mathrm{~mm}$ with a power of $80 \%$ and significance of $95 \%(a=.05)$.

To determine if tumor location affected the accuracy of the Explorer MIL system, the data were also examined based on Couinaud segment. These data are summarized in Table 4 . Comparing the 2 modalities, there was no statistically significant difference $(P=.212)$ between the tip-to-boundary distances as a function of Couinaud segment. Since the tip-toboundary measurements were not normally distributed, they were compared using the Kruskal-Wallis 1-way analysis of variance (ANOVA) on ranks where the median difference between the groups was hypothesized to be $0 \mathrm{~mm}$.

Similarly, there was no statistically significant difference between the tip-to-center distances as a function of Couinaud segment $(P=.105)$. This was determined using 1-way ANOVA, where the hypothesized mean difference between the groups was $0 \mathrm{~mm}$, based on the results of the normality $(P=.274)$ and equal variance $(P=.056)$ tests. Considering that the equality of variance assumption was somewhat questionable, an additional statistical comparison was 
performed using the Kruskal-Wallis 1-way ANOVA on ranks where the hypothesized median difference between the group was $0 \mathrm{~mm}$. This analysis also showed no statistically significant difference between the Angiodynamics StarBurst Xli-enhanced RFA system and the Covidien Evident MWA system $(P=.105)$.

\section{Discussion}

Analysis of the data collected in the clinical evaluation of the Explorer MIL system indicates that the guidance information provided by the system correlates well with information provided by iUS. Specifically, there was no statistically significant difference between the 2 modalities when measuring the distance from the probe tip to the tumor boundary. Furthermore, the mean absolute difference in the measurements is within the clinically accepted range of accuracy $(5 \mathrm{~mm}$ to $1 \mathrm{~cm}$ ) identified in the literature for laparoscopic ablation procedures. ${ }^{9-11}$ There was also no statistically significant difference found between the tip-to-center distance measurements made for the 2 guidance modalities, when a hypothesized mean difference of $5 \mathrm{~mm}$ is used in the statistical comparison. However, the mean absolute difference in the tip-to-center distance measurements was $8.57 \mathrm{~mm}$, which is greater than $5 \mathrm{~mm}$, the lower end of the clinically acceptable range. It should be noted that there is more error associated with estimating the location of the tumor center using $2 \mathrm{D}$ iUS images.

While there is a correlation between the data acquired from the 2 modalities, it is important to recall that a number of sources of confounding error exist. Because of the fact that the iUS image is 2D and the Explorer MIL system makes measurements in 3D, direct comparison of the 2 sets of distance measurements is complicated. Specifically, the tumor center and closest tumor boundary as identified on 2D iUS images may not correspond to those identified on the preoperative images used by the Explorer MIL system. Furthermore, the ability to accurately localize the tip of the ablation probe in the iUS image assumes that the probe is held directly "in-plane" with the iUS transducer. If this is not done, the tip identified in the iUS image may not correspond to the true tip of the probe. It should be noted that overall the tip-to-tumor boundary distance is subject to less bias from these confounding error sources.

In addition to the difficulties in making direct comparisons between the measurements obtained from the 2 modalities, it should be kept in mind that the distance measurements made from the tip of the ablation probe to the center of the target tumor should not be interpreted as a measure of the accuracy. In most cases, the tip of the ablation probe is not intended to be placed at the center of the target lesion. ${ }^{12}$ In procedures involving the Covidien Evident MWA system, multiple antennae are often placed around the periphery of the target tumor to produce the desired ablation zone. When using the Angiodynamics StarBurst Xli-enhanced RFA system, the goal is to place the tip of the probe in line with the center of the tumor but approximately $5 \mathrm{~mm}$ from the tumor boundary in order to completely ablate the tumor once the tines are deployed (see Figure 2). Given the extreme variability in the intended placement of the tip of the ablation probe it is difficult to accurately determine an absolute error in probe placement based on distances to the target tumor boundary or 
center. Given these limitations, the direct distance measurements depicted in Figure 4 represent the best comparison possible from the data acquired in the clinical setting.

In addition to the direct paired comparison of the distance measurements made from the 2 guidance modalities, statistical comparisons were performed to evaluate the impact of lesion location. Evaluation of the data summarized in Table 4 indicates that there is no statistically significant difference between the measurements, when analyzed based on Couinaud segment location of the target tumor.

One of the biggest sources of potential error in the Explorer MIL system is flexing of the ablation probe. The system optically tracks the Pathfinder Tracer array attached to the handle of the instrument and based on this calculates where the tip of the instrument is predicted to be. Any deflection of the probe has the potential to create an error in the calculated probe tip position. Presumably this error would be less in Covidien Evident MWA system, which is a stiffer probe; however, in this series there was no statistically significant impact based on the ablation technology used.

In conclusion, the initial clinical experience with the Explorer MIL system suggests that the device is a promising tool in providing supplemental 3D guidance information during laparoscopic ablation procedures. Although the comparison with iUS was limited in some respects, the guidance information provided by the Explorer MIL device is statistically equivalent with that provided by laparoscopic iUS. Future work will focus on the incorporation of tracked laparoscopic iUS within the system similar to that reported by Kingham et $\mathrm{al}^{2}$ for open hepatic procedures.

\section{Acknowledgments}

The authors would like to extend thanks to the support and operating room staff at Providence Portland Medical Center and the University of North Carolina Hospital for logistical assistance in performing the study. Yue-Yun To, RN, BS, OCN, and Maria Cassera, BS, were instrumental in coordinating the trial at Providence Portland Medical Center. Valorie Buchholz, RN, and Courtney Smith were instrumental in trial coordination at the University of North Carolina. Many of the algorithms and visualization tools used in this work were developed using the Visualization Toolkit (http://www.vtk.org).

\section{Funding}

The author(s) disclosed receipt of the following financial support for the research, authorship, and/or publication of this article: The work presented in this article was supported in part by grant number R01CA162477 provided by the National Institutes of Health.

\section{References}

1. Cash DM, Miga MI, Glasgow SC, et al. Concepts and preliminary data toward the realization of image-guided liver surgery. J Gastrointest Surg. 2007; 11:844-859. [PubMed: 17458587]

2. Kingham TP, Scherer MA, Neese BW, Clements LW, Stefansic JD, Jarnagin WR. Image-guided liver surgery: intraoperative projection of computed tomography images utilizing tracked ultrasound. HPB (Oxford). 2012; 14:594-603. [PubMed: 22882196]

3. Lu MD, Xu HX, Xie XY, et al. Percutaneous microwave and radiofrequency ablation for hepatocellular carcinoma: a retrospective comparative study. J Gastroenterol. 2005; 40:1054-1060. [PubMed: 16322950]

4. Poon RT, Fan ST, Tsang FH, Wong J. Locoregional therapies for hepatocellular carcinoma: a critical review from the surgeon's perspective. Ann Surg. 2002; 235:466-486. [PubMed: 11923602] 
5. Wong J, Lee KF, Yu SC, et al. Percutaneous radiofrequency ablation versus surgical radiofrequency ablation for malignant liver tumours: the long-term results. HPB (Oxford). 2013; 15:595-601. [PubMed: 23458320]

6. Simo KA, Sereika SE, Newton KN, Gerber DA. Laparoscopic-assisted microwave ablation for hepatocellular carcinoma: safety and efficacy in comparison with radiofrequency ablation. J Surg Oncol. 2011; 104:822-829. [PubMed: 21520094]

7. Irshad A, Anis M, Ackerman SJ. Current role of ultrasound in chronic liver disease: surveillance, diagnosis and management of hepatic neoplasms. Curr Probl Diagn Radiol. 2012; 41:43-51. [PubMed: 22285001]

8. Clements LW, Chapman WC, Dawant BM, Galloway RL Jr, Miga MI. Robust surface registration using salient anatomical features for image-guided liver surgery: algorithm and validation. Med Phys. 2008; 35:2528-2540. [PubMed: 18649486]

9. Kuang M, Lu MD, Xie XY, et al. Liver cancer: increased microwave delivery to ablation zone with cooled-shaft antenna—experimental and clinical studies. Radiology. 2007; 242:914-924. [PubMed: 17229876]

10. Goldberg SN, Gazelle GS, Compton CC, Mueller PR, Tanabe KK. Treatment of intrahepatic malignancy with radiofrequency ablation: radiologic-pathologic correlation. Cancer. 2000; 88:2452-2463. [PubMed: 10861420]

11. Dodd GD 3rd, Frank MS, Aribandi M, Chopra S, Chintapalli KN. Radiofrequency thermal ablation: computer analysis of the size of the thermal injury created by overlapping ablations. AJR Am J Roentgenol. 2001; 177:777-782. [PubMed: 11566672]

12. Brace CL. Radiofrequency and microwave ablation of the liver, lung, kidney, and bone: what are the differences? Curr Probl Diagn Radiol. 2009; 38:135-143. [PubMed: 19298912] 

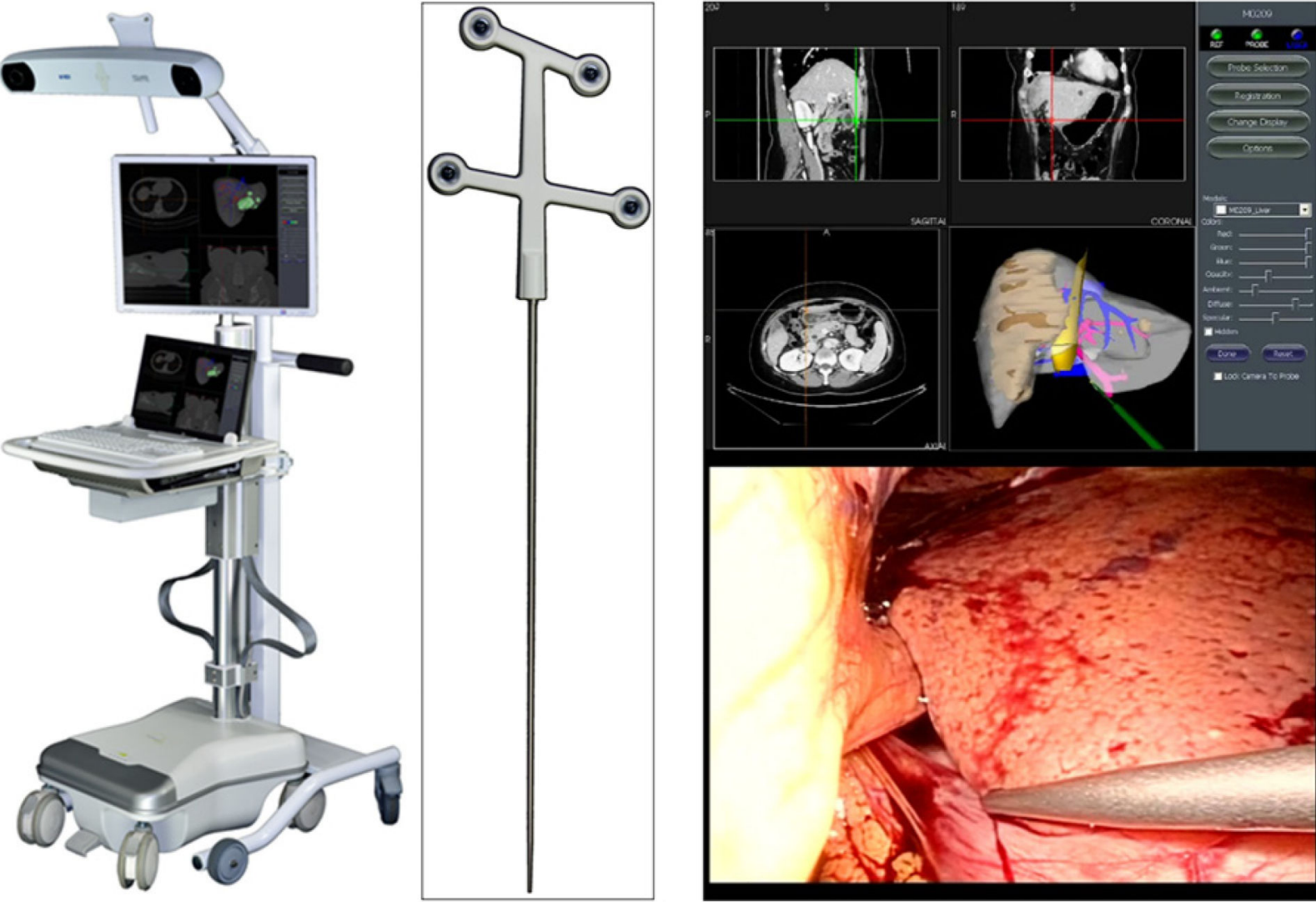

Figure 1.

Image of the Explorer Minimally Invasive Liver (MIL) device with the tracking system cart and the tracked laparoscopic stylus (left). Screen captures of the guidance system (top right) and the corresponding laparoscopic view (bottom right) show the stylus being used; it is shown as green in the virtual model. 

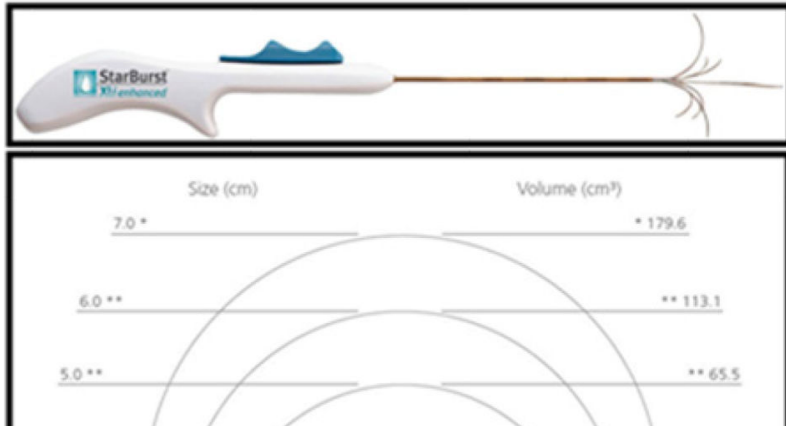

$40 \cdots$

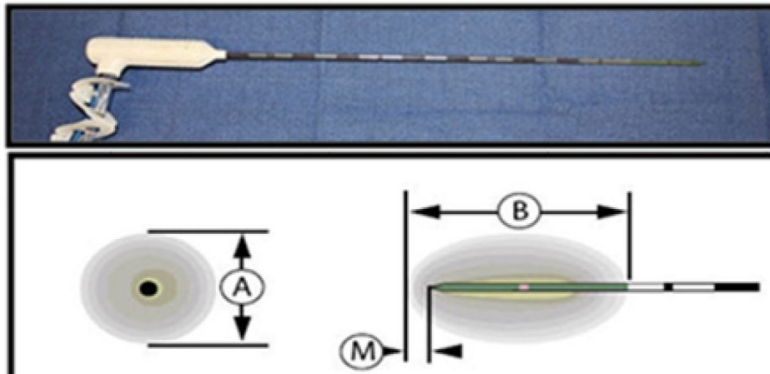

\begin{tabular}{l|lll} 
& $30 \mathrm{~W}$ & \multicolumn{2}{c}{$45 \mathrm{~W}$} \\
\hline & $10: 00$ & $5: 00$ & $10: 00$ \\
\hline A & $2.2 \mathrm{~cm}$ & $2.3 \mathrm{~cm}$ & $2.8 \mathrm{~cm}$ \\
\hline B & $3.3 \mathrm{~cm}$ & $3.2 \mathrm{~cm}$ & $4.0 \mathrm{~cm}$ \\
\hline$M$ & $0.5 \mathrm{~cm}$ & $0.6 \mathrm{~cm}$ & $0.7 \mathrm{~cm}$ \\
\hline & $9 \mathrm{~cm}^{3}$ & $9 \mathrm{~cm}^{3}$ & $16 \mathrm{~cm}^{3}$
\end{tabular}

Figure 2.

Images of the Angiodynamics StarBurst Xli-Enhanced radiofrequency ablation system (left) and the Covidien Evident microwave ablation system (right) along with the manufacturer specifications of the predicted ablation zones. 


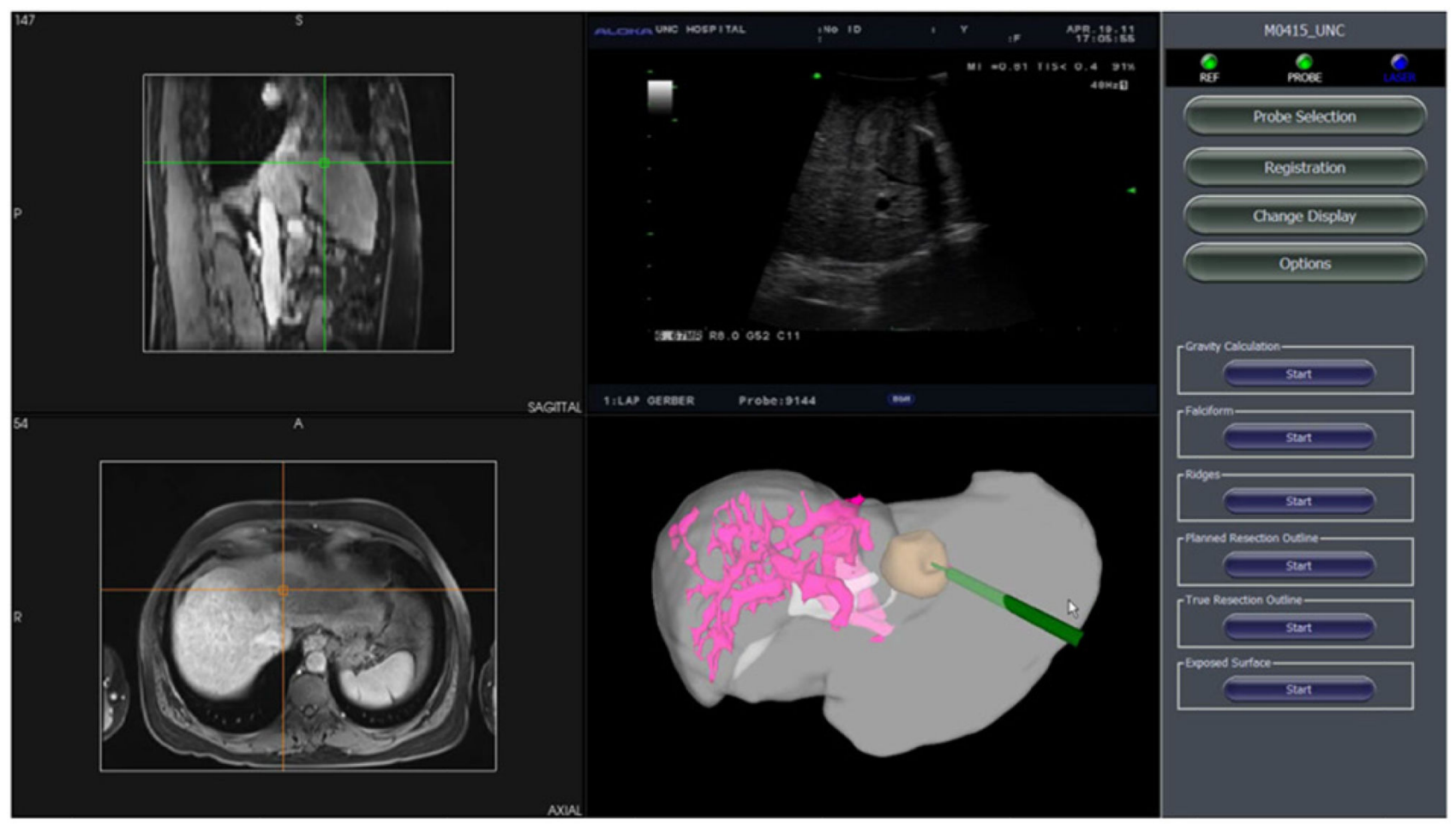

Figure 3.

Screen capture of the software display of the Explorer Minimally Invasive Liver system during a laparoscopic ablation procedure. The display shows tracked location of the tip of the ablation probe on the sagittal (top left quadrant) and axial (bottom left quadrant) slice of the magnetic resonance image. In the bottom right quadrant, the 3-dimensional virtual model is shown with the tumor in brown and the ablation probe in green. The streaming video feed from the intraoperative ultrasound is displayed in the top right quadrant. 

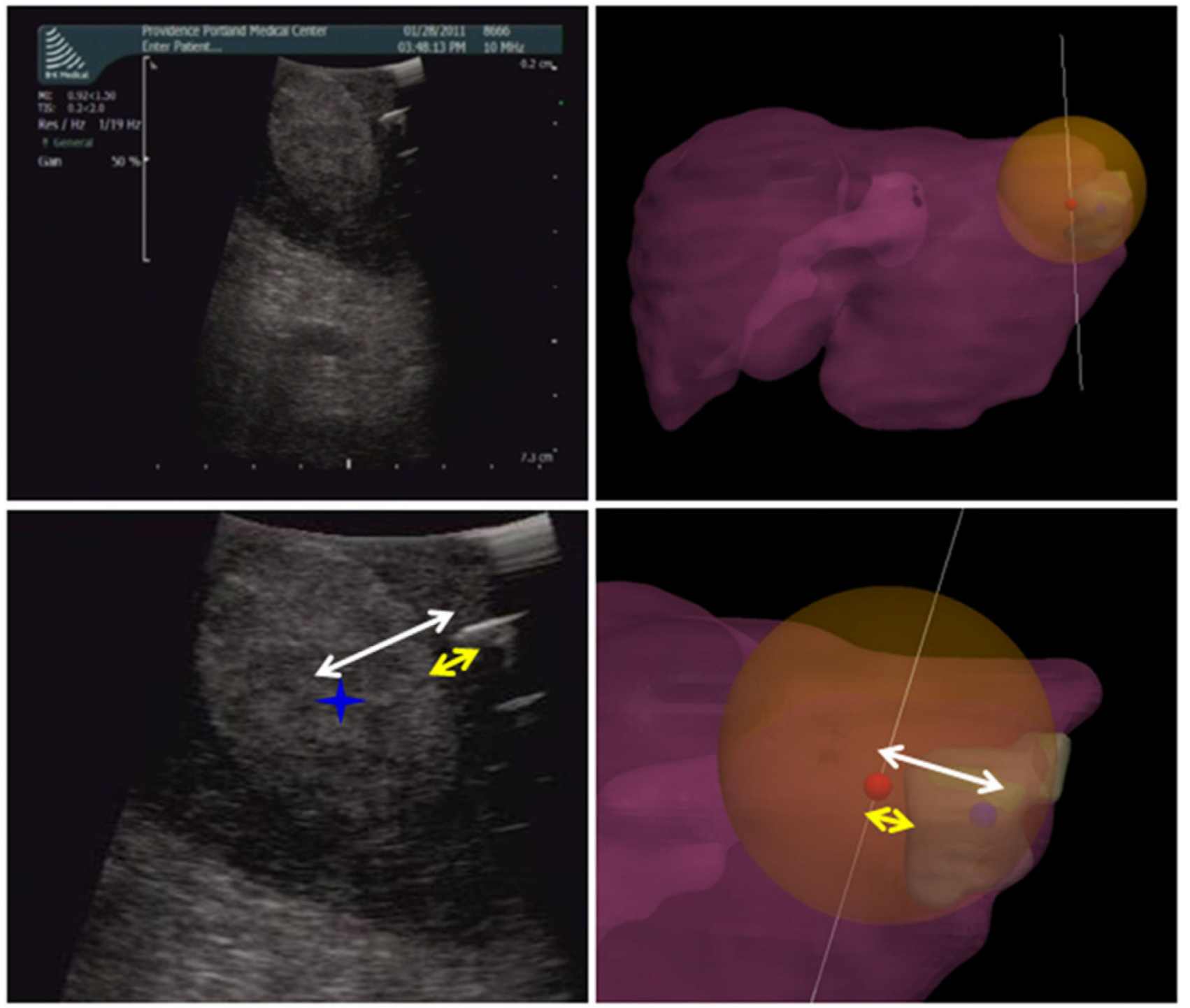

Figure 4.

Example of the measurements obtained from intraoperative ultrasound (iUS; left) and Explorer Minimally Invasive Liver (MIL) system (right) are shown. The distance from the probe tip to the tumor boundary (yellow arrow) and the distance from the probe tip to tumor center (white arrow) are illustrated. In the iUS image, the tumor center is identified by a blue star. The virtual model from the Explorer MIL system shows the recorded location of the probe tip (red sphere), the tumor being targeted (gray), the tumor center (blue sphere), and projected ablation zone (orange). 


\section{Table 1}

Summary of the Demographics of the Patients Accrued in the Evaluation of the Explorer Minimally Invasive Liver System.

\begin{tabular}{lc}
\hline Total patients & 27 \\
Sex & 23 \\
Male & 4 \\
Female & $60.1 \pm 6.9$ \\
Age in years & \\
Preoperative imaging & 11 \\
Computed tomography & 16 \\
Magnetic resonance & 31 \\
Total lesions & \\
Lesion location & 0 \\
I & 6 \\
II & 0 \\
III & 7 \\
IV & 3 \\
V & 3 \\
VI & 3 \\
VII & 5 \\
VIII & 7 \\
Ablation modality & \\
Microwave & \\
Radiofrequency & \\
Pathology & 16 \\
Hepatocellular carcinoma & \\
Metastatic colorectal carcinoma & 8 \\
Other metastases & \\
\hline & \\
\hline
\end{tabular}



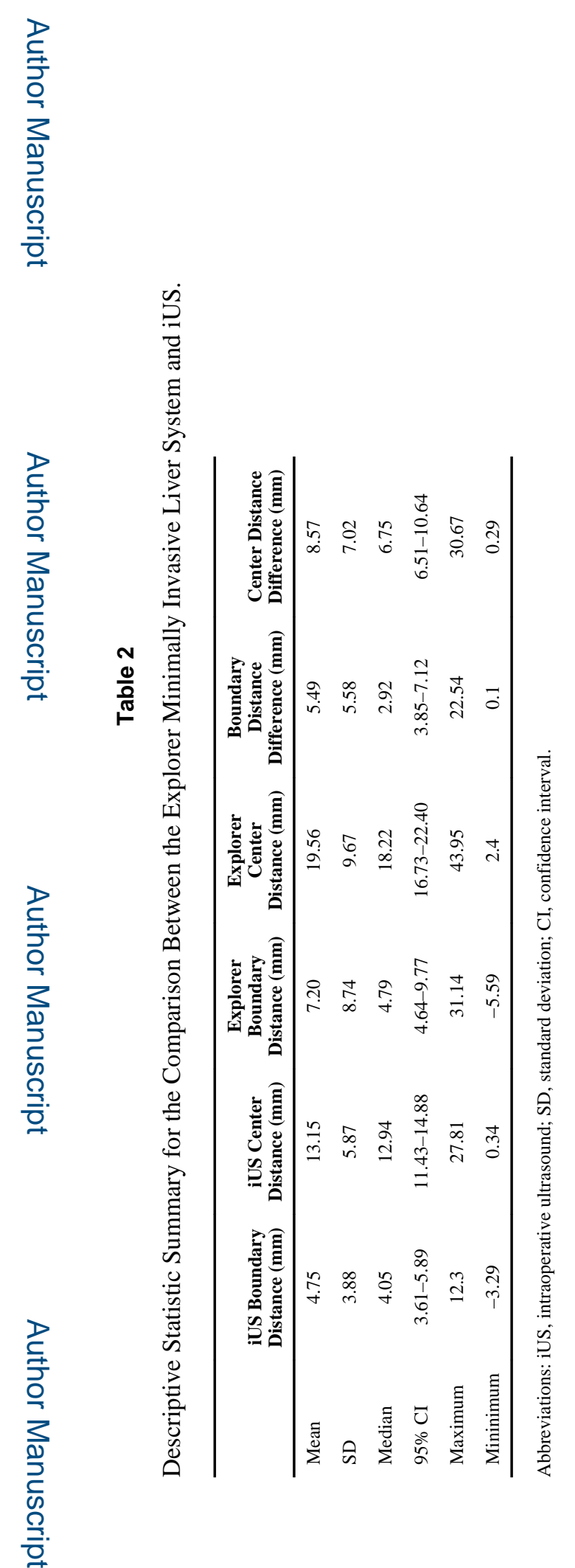

Surg Innov. Author manuscript; available in PMC 2015 April 08. 
Table 3

Summary of the Statistical Analysis Performed to Compare the Distance Measurements Made Using iUS and the Explorer Minimally Invasive Liver System.

\begin{tabular}{lcc}
\hline & Normality & Paired $t$ Test/WSR Test \\
\hline Tip-boundary distance $\left(\right.$ WSR test, $\left.M_{\text {diff }}=0 \mathrm{~mm}\right)$ & Failed $(P<.05)$ & Accept $\mathrm{H}_{0}(P=.06)$ \\
Tip-center distance $\left(t\right.$ test, $\left.\mu_{\text {diff }}=0 \mathrm{~mm}\right)$ & Passed $(P=.71)$ & Reject $\mathrm{H}_{0}(P<.001)$ \\
Tip-center distance $\left(t\right.$ test, $\left.\mu_{\text {diff }}=5 \mathrm{~mm}\right)$ & Passed $(P=.71)$ & Accept $\mathrm{H}_{0}(P=.29)$ \\
\hline
\end{tabular}

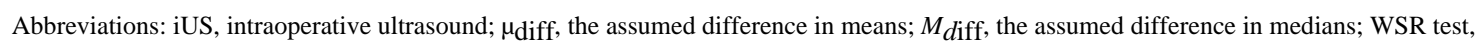
Wilcoxon signed rank test. 


\section{Table 4}

Summary of the Absolute Differences Between iUS and the Explorer Minimally Invasive Liver System Analyzed by Couinaud Segment.

\begin{tabular}{lcc}
\hline Couinaud Segment & $\begin{array}{c}\text { Tip-Boundary Difference } \\
(\mathbf{m m}, \boldsymbol{\mu} \pm \mathbf{9 5} \% \mathbf{C I})\end{array}$ & $\begin{array}{c}\text { Tip-Center Difference } \\
(\mathbf{m m}, \boldsymbol{\mu} \pm \mathbf{9 5} \% \mathbf{C I})\end{array}$ \\
\hline $2(\mathrm{~N}=8)$ & $2.11 \pm 1.29$ & $4.83 \pm 2.41$ \\
$3(\mathrm{~N}=0)$ & $\mathrm{N} / \mathrm{A}$ & $\mathrm{N} / \mathrm{A}$ \\
$4(\mathrm{~N}=10)$ & $5.83 \pm 3.29$ & $7.42 \pm 3.51$ \\
$5(\mathrm{~N}=4)$ & $2.99 \pm 1.54$ & $5.44 \pm 3.54$ \\
$6(\mathrm{~N}=6)$ & $8.20 \pm 6.21$ & $14.62 \pm 9.12$ \\
$7(\mathrm{~N}=7)$ & $5.91 \pm 5.02$ & $11.16 \pm 5.40$ \\
$8(\mathrm{~N}=11)$ & $6.72 \pm 3.69$ & $9.26 \pm 3.69$ \\
\hline
\end{tabular}

Abbreviations: iUS, intraoperative ultrasound; CI, confidence interval; N/A, not applicable. 